DOI: 10.17707/AgricultForest.62.2.08

\author{
Miloje ŠUNDIĆ, Ryszard HAITLINGER, \\ Ivana JOVIČIĆ, Olivera PETROVIĆ-OBRADOVIĆ ${ }^{I}$
}

\title{
NEW HOST DATA FOR TERRESTRIAL PARASITENGONA OF SERBIA WITH NOTE ON Allothrombium clavatum Saboori, Pešić \& Hakimitabar, 2010
}

\begin{abstract}
SUMMARY
Allothrombium clavatum Saboori, Pešić \& Hakimitabar, 2010 was described from Montenegro, based on two damaged specimens. This species was reported for the first time from Serbia on aphid Aphis craccivora. In this paper, new host and metric data are given. Further, on 7 localities, in different parts of Serbia, larvae of three trombidiid mite species on A. craccivora have been found.

Keywords: Allothrombium clavatum, new metric data, Aphis craccivora, Serbia.
\end{abstract}

\section{INTRODUCTION}

Fauna of terrestrial Parasitengona of Serbia is poorly known. Hitherto only thirteen species of this fauna have been reported from Serbia: Allothrombium fuliginosum (Hermann, 1804); Abrolophus stanislavae Haitlinger, 1986; A. quisquiliarus (Hermann, 1804); Balaustium nikae Haitlinger, 1996; Erythraeus (Zaracarus) budapestensis Fain \& Ripka, 1998; Eutrombidium trigonum (Hermann, 1804); Microtrombidium parvissimum Gabryś, 1999; Charletonia cardinalis (C. L. Koch, 1837) (Mąkol \& Wohltmann 2012, 2013; Haitlinger 2007, 2012) and Abrolophus montenegrinus Saboori, Šundić \& Pešić, 2012; Leptus eslamizadehi Saboori, 2002; Erythraeus (Erythraeus) mariolae Haitlinger, 1994; Charletonia krendowskyi Feider, 1954; Erythraeus (Erythraeus) serbicus Šundić, Haitlinger, Petanović, Jovičić \& Hakimitabar 2015 (Šundić et al., 2015).

Allothrombium clavatum Saboori, Pešić \& Hakimitabar, 2010 is new record of fauna terrestrial Parasitengona of Serbia. This species was described from Montenegro, based on two damaged specimens by Saboori, et al., 2010. Scutum was drawn without PL setae and sensillae (S) therefore figure of scutum, new host and metric data are given (Fig. 1, Table 1). Also trombidiid species found on Aphis craccivora Koch, 1854 in Serbia are listed.

\footnotetext{
${ }^{1}$ Miloje Šundić,(corresponding author: miloje@t-com.me) Department of Biology, Faculty of Sciences, University of Montenegro, Cetinjski put b. b., 81000Podgorica, MONTENEGRO, Ryszard Haitlinge, Department of Invertebrate Systematics and Ecology, Institute of Biology, Wrocław University of Environmental and Life Sciences, Kożuchowska 5B, 51-631 Wrocław, POLAND, Ivana JOVIČIĆ, Institut of Phytomedicine, Faculty of Agriculture, University of Belgrade, Nemanjina 6, 11080 Belgrade, Zemun. SERBIA, Olivera Petrović-Obradović, Institut of Phytomedicine, Faculty of Agriculture, University of Belgrade, Nemanjina 6, 11080 Belgrade, Zemun. SERBIA

Notes: The authors declare that they have no conflicts of interest. Authorship Form signed online.
} 


\section{MATERIAL AND METHODS}

Total two larvae of mites Allothrombium clavatum were collected in Serbia from host A. craccivora (Hemiptera: Aphididae) during the June 2013. The specimens of A. craccivora were collected in Serbia from alfalfa (Medicago sativa L.). Sampling of aphids and larvae of mites was done at different intervals for four years (2011-2014). Aphids were collected directly from plant stems and placed in plastic tubes in $70 \%$ ethanol. Samples were labelled and transferred to the laboratory for identification based on morphological characters using a stereoscopic microscope (Bio-Optica, Type: 1000). In the laboratory, mates were separated from the aphids and preserved in 75\% alcohol, cleared in Nessbitt's solution and mounted in Faure medium (Walter \& Krantz, 2009). All measurements are given in micrometers $(\mu \mathrm{m})$ and calculated using a Carl Zeiss Axioscope A1 microscope, and Carl Zeiss Axio Imager A2 with differential interference contrast and phase contrast. The terminology and abbreviations follow Makol (2007).

\section{RESULTS AND DISCUSSION}

Allothrombium clavatum Saboori, Pešić \& Hakimitabar, 2010: 515-519, figs: $1-6$.

Material: 2 larvae, Serbia, locality Cerje, Ušće, 29 June 2013.

Distribution: Montenegro, Serbia.

Polyphagous species A. craccivora is a widely distributed species that has been reported as a pest of many crops on several continents (Blackman and Eastop, 2000, Berberet et al., 2009), but its origins are clearly in Europe (Blackman and Eastop, 2007). In Serbia, this species has been reported on about 30 host plants (Petrović-Obradović, 2003). Aphis craccivora has been recorded as pests of alfalfa in this country (Jovičić et al., 2014).

On 7 localities, in different parts of Serbia, we have found larvae of mites on this aphid (Table 2). Total 33 mites were found on 21 aphids, among them 2 mites Allothrombium clavatum, 5 mites Erythraeus (E.) serbicus and 26 mites Allothrombium fuliginosum (Hermann, 1804). Most captured specimens of $A$. craccivora were attached by one mite. Maximum 3 mites Erythraeus (E.) serbicus were attached on this host. The most of mites were attached on wingless adults. Most of the mites were located above the thorax of the aphids. Only a few individuals were attached on the abdomen of A. craccivora. Mites were present on this aphid in period June-August. The first individuals of mites were collected at the beginning of June at Central Serbia (Belosavci and Donja Šatornja), (Table 2).

\section{Remarks}

Morphological variability A. clavatum in specimens from Serbia is distinctly differs of A. clavatum specimens from Montenegro, especially in metric data of SD, W, AA, ASB, PSB, MA (Table 1). Anterior border of scutum rounded, posterior border of scutum is slightly 3 lobed (in original description posterior border of scutum is more prominent 3 lobed) (Fig.1). 
Table 1. Metric data for Allothrombium clavatum Sabori, Pešić \& Hakimitabar 2010. Hholotype, P-paratype, specimens from Montenegro; S1, S2-specimens from Serbia

\begin{tabular}{|c|c|c|c|c|c|c|c|c|c|}
\hline & H & P & S1 & S2 & & Holotype & Paratype & S1 & S2 \\
\hline IL & 329 & 247 & 848 & 630 & or & 6 & - & 6 & 7 \\
\hline IW & 235 & 272 & 548 & 503 & $b s$ & 7 & - & 8 & 9 \\
\hline SD & 64 & 57 & 84 & 85 & Ta I (L) & 40 & 50 & 54 & 58 \\
\hline W & 62 & 62 & 83 & 82 & Ta I (H) & 20 & 22 & 22 & 21 \\
\hline AW & 53 & 45 & 54 & 55 & Ti I & 42 & 46 & 35 & 40 \\
\hline PW & 65 & - & 67 & 70 & Ge I & 25 & 30 & 22 & 27 \\
\hline AA & 38 & - & 47 & 42 & Fe I & 42 & 47 & 43 & 43 \\
\hline SB & 37 & 32 & 35 & 38 & Tr I & 32 & 35 & 28 & 32 \\
\hline ASB & 31 & - & 46 & 51 & Cx I & 50 & 45 & 53 & 52 \\
\hline PSB & 33 & - & 42 & 40 & Leg I & 231 & 253 & 235 & 252 \\
\hline MA & 17 & - & 33 & 33 & Ta I (L) & 45 & 43 & 45 & 51 \\
\hline AP & 26 & - & 22 & 29 & Ta II (H) & 19 & 17 & 20 & 19 \\
\hline AL & 28 & 28 & 28 & 33 & Ti II & 41 & 37 & 33 & 35 \\
\hline PL & - & 33 & 33 & 43 & Ge II & 23 & 25 & 20 & 22 \\
\hline AM & 32 & 35 & 31 & 31 & Fe II & 41 & 40 & 41 & 37 \\
\hline S & - & 35 & 37 & 45 & Tr II & 34 & 32 & 28 & 23 \\
\hline LSS & 53 & 57 & 65 & 59 & Cx II & 50 & 52 & 51 & 45 \\
\hline HS & 22 & 25 & 23 & 21 & Leg II & 234 & 229 & 218 & 213 \\
\hline SL & - & 31 & 32 & 33 & Ta III (L) & 45 & 50 & 50 & 54 \\
\hline SS & 25 & 28 & 24 & 25 & Ta III (H) & 15 & 16 & 18 & 16 \\
\hline DS & $22-30$ & $25-28$ & $26-29$ & $27-33$ & Ti III & 44 & 47 & 41 & 42 \\
\hline PDS & 20 & 22 & 20 & 21 & Ge III & 25 & 26 & 22 & 25 \\
\hline $1 a$ & 32 & 30 & 33 & 35 & Fe III & 37 & 40 & 46 & 38 \\
\hline$I b$ & 27 & 29 & 30 & 32 & Tr III & 37 & 40 & 25 & 28 \\
\hline $2 b$ & 32 & 35 & 35 & 30 & Cx III & 47 & 50 & 52 & 48 \\
\hline $3 a$ & 31 & 30 & 26 & 26 & Leg III & 235 & 235 & 236 & 235 \\
\hline $3 b$ & 32 & 35 & 25 & 34 & IP & 700 & 735 & 689 & 700 \\
\hline
\end{tabular}

Leg setal formula for specimens from Serbia: Leg I: Ta $1 \omega, 2 \zeta, 1 \varepsilon, 13 \mathrm{n}$; Ti $2 \varphi$,

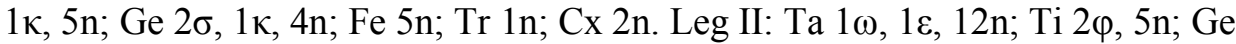
$2 \sigma, 1 \kappa, 3 n ;$ Fe 4n; Tr 1n; Cx 1n. Leg III: Ta 12n; Ti 5n; Ge 2o, 3n; Fe 4n; Tr 1n; Cx 1n.

$\mathrm{NDV}=24+6=30$

Metric data of species from Montenegro and Serbia are given in Table 1. 


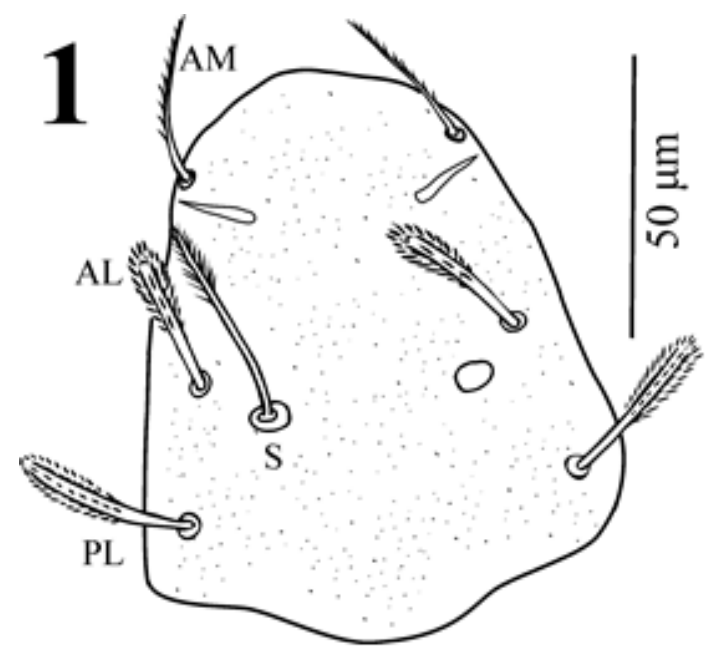

Figure 1. Allothrombium clavatum Saboori, Pešić \& Hakimitabar 2010, Scutum

Table 2. Parasitengonae mites found on Aphis craccivora in Serbia

\begin{tabular}{|c|c|c|c|c|}
\hline Locality name & $\begin{array}{l}\text { Geographic } \\
\text { coordinates }\end{array}$ & Mites & Host & $\begin{array}{c}\text { Number of } \\
\text { mites }\end{array}$ \\
\hline $\begin{array}{c}\text { Belosavci, } \\
\text { Topola }\end{array}$ & $\begin{array}{l}44^{\circ} 20^{\prime} 31^{\prime \prime}, \mathrm{N} \\
20^{\circ} 40^{\prime} 58^{\prime} \mathrm{E}\end{array}$ & $\begin{array}{c}\text { Allothrombium } \\
\text { fuliginosum }\end{array}$ & A. craccivora & 2 \\
\hline $\begin{array}{c}\text { Braničevo, } \\
\text { Golubac }\end{array}$ & $\begin{array}{l}44^{\circ} 41^{\prime} 54^{\prime}{ }^{\prime} \mathrm{N} \\
20^{\circ} 32^{\prime} 29^{\prime} \mathrm{E}\end{array}$ & $\begin{array}{l}\text { Allothrombium } \\
\text { fuliginosum }\end{array}$ & A. craccivora & 4 \\
\hline Cerje, Ušće & $\begin{array}{l}43^{\circ} 29^{\prime} 59^{\prime \prime} \mathrm{N} \\
20^{\circ} 36^{\prime} 50^{\prime \prime} \mathrm{E}\end{array}$ & $\begin{array}{l}\text { Allothrombium } \\
\text { clavatum }\end{array}$ & A. craccivora & 2 \\
\hline $\begin{array}{c}\text { Donja Šatornja, } \\
\text { Topola }\end{array}$ & $\begin{array}{l}41^{\circ} 11^{\prime} 11^{\prime \prime} \mathrm{N} \\
20^{\circ} 33^{\prime} 09^{\prime}, \mathrm{E}\end{array}$ & $\begin{array}{l}\text { Allothrombium } \\
\text { fuliginosum }\end{array}$ & A. craccivora & 5 \\
\hline $\begin{array}{l}\text { Kotraža, } \\
\text { Lučani }\end{array}$ & $\begin{array}{l}43^{\circ} 41^{\prime} 48^{\prime \prime}, \mathrm{N} \\
20^{\circ} 14^{\prime} 45^{\prime \prime} \mathrm{E}\end{array}$ & $\begin{array}{l}\text { Allothrombium } \\
\text { fuliginosum }\end{array}$ & A. craccivora & 6 \\
\hline Ovča, Beograd & $\begin{array}{l}44^{\circ} 52^{\prime} 49^{\prime \prime} \mathrm{N} \\
20^{\circ} 32^{\prime} 13^{\prime \prime} \mathrm{E}\end{array}$ & $\begin{array}{l}\text { Allothrombium } \\
\text { fuliginosum }\end{array}$ & A. craccivora & 3 \\
\hline $\begin{array}{l}\text { Rusko selo, } \\
\text { Kikinda }\end{array}$ & $\begin{array}{l}45^{\circ} 45^{\prime} 16^{\prime \prime}, \mathrm{N} \\
20^{\circ} 33^{\prime} 47^{\prime} \mathrm{E}\end{array}$ & Erythraeus serbicus & A. craccivora & 5 \\
\hline $\begin{array}{c}\text { Rusko selo, } \\
\text { Kikinda }\end{array}$ & $\begin{array}{l}45^{\circ} 45^{\prime} 16^{\prime \prime} \mathrm{N} \\
20^{\circ} 33^{\prime} 47^{\prime} \mathrm{E}\end{array}$ & $\begin{array}{l}\text { Allothrombium } \\
\text { fuliginosum }\end{array}$ & A. craccivora & 6 \\
\hline
\end{tabular}




\section{CONCLUSIONS}

Allothrombium clavatum is new record of fauna terrestrial Parasitengona of Serbia. Morphological variability A. clavatum in specimens from Serbia is distinctly differs of A. clavatum specimens from Montenegro. Larvae of three mite species on A. craccivora were found: Allothrombium clavatum, Erythraeus (E.) serbicus and Allothrombium fuliginosum. This is the first investigation of the occurrence of trombidiid mites on this aphid in Serbia.

\section{REFERENCES}

Berberet R. C., K. L. Giles, A. A. Zarrabi \& M. E. Payton 2009. Development, reproduction, and within-plant infestation patterns of Aphis craccivora (Homoptera: Aphididae) on alfalfa. - Environmental entomology, 38(6): 17651771

Blackman R. L. \& V. F. Eastop 2000. Aphids on the World's Crops. An Identification and Information Guide. Second Edition. Chichester UK: John Wiley \& Sons. 476 pp.

Blackman R. L. \& V. F. Eastop 2007. Taxonomic Issues. In: Aphids as Crop Pests, Edited by. Van Emden, H. F. and Harrington, R. CAB International, 1-29.

Haitlinger R. 2007. New species and records of mites (Acari, Prostigmata: Erythraeidae, Trombidiidae, Eutrombidiidae) from the Balkan Peninsula. Biologia. 62: 67-77.

Haitlinger R. 2012. New records of mites (Acari: Prostigmata: Erythraeidae, Trombi-diidae) from Albania, Macedonia, Montenegro and Serbia, with a description of Erythraeus (Erythraeus) albanicus sp. nov. Systematic \& Applied Acarology, 17(3): 339-345.

Jovičić I., A Vučetić, Ž Tomanović \& O. Petrović-Obradović 2014. Natural enemies of alfalfa aphids in Serbia. VII Congres on plant protection, Zlatibor, Serbia, 119120.

Makol J. 2007. Generic level review and phylogeny of Trombidiidae and Podothrombiidae (Acari: Actinotrichida: Trombidioidea) of the world. Annales Zoologici, 57 (1): 1-194.

Mąkol J. \& A. Wohltmann 2012. An annotated checklist of terrestrial Parasitengona (Actinotrichida: Prostigmata) of the world, excluding Trombiculidae and Walchiidae. Annales Zoologici, 62 (3): 359-562.

Mąkol J. \& A. Wohltmann 2013. Corrections and additions to the checklist of terrestrial Parasitengona (Actinotrichida: Prostigmata) of the world, excluding Trombiculidae and Walchiidae. Annales Zoologici, 63 (1): 15-27.

Petrović - Obradović O. 2003. Biljne vaši (Homoptera: Aphididae) Srbije. Poljoprivredni fakultet Univerziteta u Beogradu (in Serbian), $153 \mathrm{pp}$.

Saboori A., Pešić V. \& M. Hakimitabar 2010. A new species of the genus Allothrombium (Acari: Trombidiidae) from Montenegro. Biologia, 65/3: 515-519, 2010.

Šundić M., Haitlinger R., Petanović R., Jovičić M. \& M. Hakimitabar 2015. A new species of Erythraeus (Erythraeus) and new records of mites (Acari: Erythraeidae) from Serbia. Biologia, 70/6: 788—796, 2015.

Walter D.E. \& G.W. Krantz 2009. Collecting, rearing, and preparing specimens, pp. 8396. In: Krantz G.W. \& Walter D.E. (eds), A Manual of Acarology, 3rd edition, Texas Tech. Univ. Press, 816 pp. ISBN-13: 978-0896726208, ISBN- 10: 0896726207. 